\title{
Linx
}

Revue des linguistes de l'université Paris X Nanterre

$13 \mid 2020$

Collocations et traditions discursives

\section{Conglomerati di lingua e immagini nel discorso on line dell'attivismo politico}

Modelli, esempi

Silvia Verdiani

\section{CpenEdition}

\section{Journals}

Edizione digitale

URL: http://journals.openedition.org/linx/3942

DOI: $10.4000 / \operatorname{linx} .3942$

ISSN: 2118-9692

Editore

Presses universitaires de Paris Nanterre

Notizia bibliografica digitale

Silvia Verdiani, «Conglomerati di lingua e immagini nel discorso on line dell'attivismo politico », Linx

[En ligne], 13 | 2020, mis en ligne le 30 mai 2020, consulté le 01 juin 2020. URL : http://

journals.openedition.org/linx/3942 ; DOI : https://doi.org/10.4000/linx.3942

Questo documento è stato generato automaticamente il 1 giugno 2020.

Département de Sciences du langage, Université Paris Ouest 


\section{Conglomerati di lingua e immagini nel discorso on line dell'attivismo politico}

Modelli, esempi

Silvia Verdiani

\section{La dimensione olistica della comunicazione digitale}

1 La ricerca di ambito linguistico dedicata alla comunicazione online ha evidenziato che la dimensione comunicativa digitale condensa in sé tratti pertinenti ad ambiti che prima dell'avvento di Internet erano considerati distanti fra loro; in particolare sembra caratterizzata da una forma di dialogicità, in cui la lingua scritta si flette in realizzazioni che sono tipiche del parlato, potenziando la sua espressività con l'introduzione di citazioni multimediali. Il testo è in generale il prodotto di un atto comunicativo complesso, un intreccio strutturato di significati verbali ed extraverbali, in buona parte basati sulle conoscenze dei parlanti e sulla loro capacità di attivarne il senso. Nella comunicazione digitale la dimensione scritta in tutte le sue forme si accompagna però sempre più spesso a quella visuale, acustica e sensoriale, estendendo in questo modo la potenzialità semantica dei messaggi. La sua analisi richiede quindi un approccio teorico di tipo interdisciplinare ${ }^{1}$ che giunga alla formulazione di nuove categorie descrittive aperte, capaci di integrare la dimensione multimodale, in tutte le sue numerose manifestazioni. Adottando una categoria recentemente introdotta da Massimo Prada (2015: 50) in relazione all'italiano potremmo dunque definire olistica la dimensione che caratterizza l'attuale comunicazione digitale, dimensione tracciabile anche in passato, seppure in misura più limitata, in tutte quelle produzioni che sovrapponevano realizzazioni di materiali semici diversi all'interno di uno stesso testo (cf. Verdiani 2019), ma che al momento attuale, nell'«era biomediatica» (cf. Censis-Ucsi 2017) in cui viviamo, costituisce uno standard sempre più diffuso. 
2 Una delle peculiarità di testi così complessi è il nuovo ruolo assunto dalla lingua, un ruolo determinante per la costituzione del significato, ma caratterizzato da una funzionalità specifica che non può prescindere dalla sua natura ibrida. Tenendo conto di questa funzionalità complessa Ulrich Schmitz (2011: 37) propone di individuare accanto agli 'atti linguistici', Sprechakte, e agli 'atti testuali', Textakte, anche delle unità minime formate da parole e immagini che definisce 'atti visuali', Bildakte. Una parte molto importante nella lettura dei messaggi multimodali la gioca infatti proprio la memoria visiva, cioè la presenza di alcune 'immagini chiave', Schlagbilder (Diekmannshenke 2011: 161) che persistono nella memoria personale e collettiva. Esse costituiscono un patrimonio culturale molto importante e consentono di trasmettere in modo puntuale e sintetico i riferimenti storici e culturali, carichi della dimensione emotiva che gli eventi hanno determinato nell'utente.

Lo scritto trasmesso nella rete è dunque una produzione molto eterogena, che talvolta è orientata verso lo scritto-scritto, talvolta verso uno scritto fortemente influenzato dal parlato, al punto che, come è emerso chiaramente in un recente studio dedicato alla comunicazione in rete pubblicato dall'Accademia della $\mathrm{Crusca}^{2}$, parlare di lingua digitale sembra ormai riduttivo e si preferisce analizzare le singole produzioni alla luce dei loro tratti specifici. Se si tiene conto della dipendenza dalle immagini e dal materiale multimediale in genere dei messaggi pubblicati in rete, infatti, la situazione sugli assi di variazione risulta piuttosto articolata (Fig. 1).

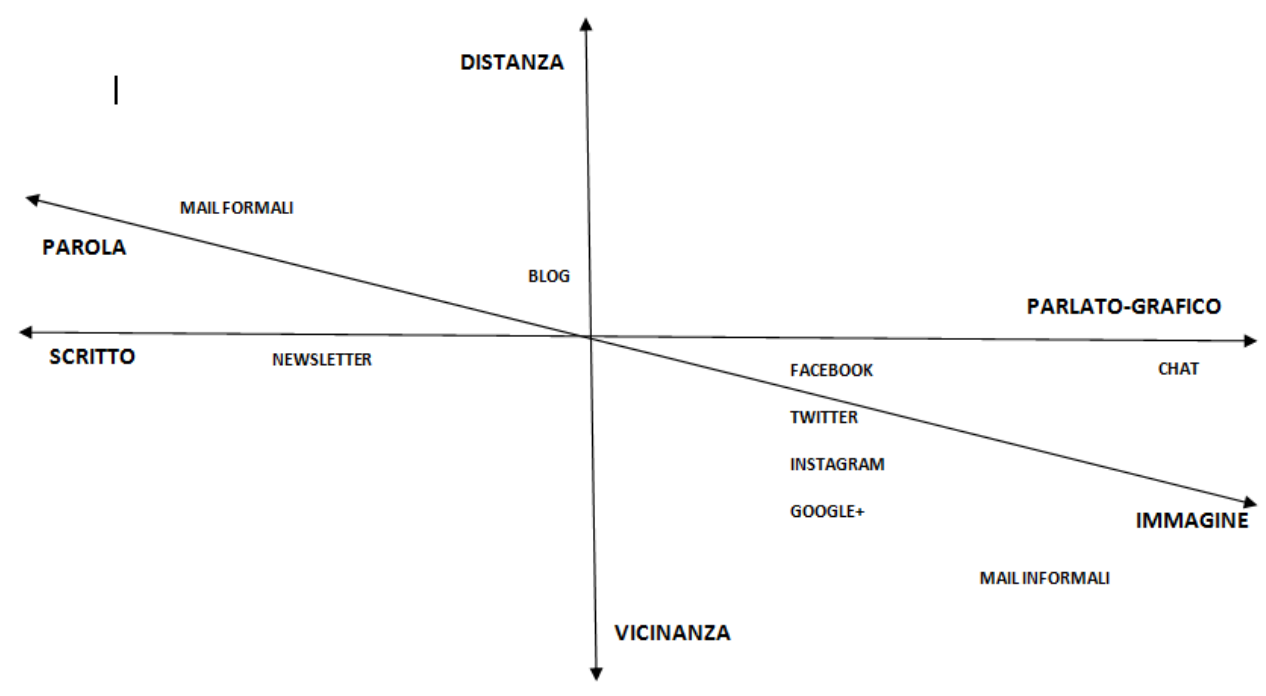

Fig. 1 (Verdiani 2016: 457)

Giungere alla descrizione della comunicazione in ambiente digitale significa dunque, in un certo senso, riconfigurare i modelli di riferimento del panorama teorico linguistico, partendo dal presupposto che ormai un'analisi che prescinda del corredo multimediale e multimodale presente nella comunicazione in rete sembra difficilmente immaginabile. Il testo verbale in rete in molti casi infatti non è autonomo, non è comprensibile se non in riferimento alle immagini a cui è associato. Questo orizzonte di ricerca emerge chiaramente già nelle scelte terminologiche operate sino ad ora nel tentativo di definire l'oggetto d'analisi dall'iconolinguistica in cui vengono sintetizzati i due punti focali della ricerca, il testo verbale e l'immagine: Text-Bild-Gemenge, Text-BildKonglomerate ${ }^{3}$, ecc. A sostenere questa prospettiva di ricerca contribuiscono però anche 
alcuni approcci teorici precedenti, per esempio la direzione di ricerca incentrata sull'analisi linguistica dell'intertestualità di Gerda Haßler o la descrizione della comunicazione multimediale di János S. Petőfi.

\section{L'analisi linguistica a partire dal testo ibrido}

5 Eugenio Coseriu sosteneva che tutto ciò che è atto linguistico, o che è una compagine di atti linguistici a esso interconnessi è un testo (1997: 31), egli riteneva che il linguaggio dovesse essere analizzato a partire dal testo reale e definiva la linguistica del testo come una linguistica a partire dal testo. L'analisi linguistica del testo consente infatti di comprenderne i diversi aspetti semantici del testo e come l'intreccio dei vari piani venga messo in scena, nella sua analisi essa attribuisce alla lingua un ruolo di primo piano, individuandone regolarità e caratteristiche. Ora, se è vero che in ambiente digitale l'oggetto d'analisi continuano a essere i nessi tra sequenze di frasi e gli altri elementi semanticamente rilevanti che costituiscono il testo, tuttavia la presenza di elementi extratestuali, che potremmo definire ad alto potenziale semantico, sembra richiedere una revisione dei parametri teorici di riferimento: i ragionamenti contestuali evocati dalla presenza di immagini e altri materiali multimediali influenzano non poco anche le scelte lessicali, sintattiche e interpuntive del testo verbale. Infatti, come osserva Angela Ferrari, i confini dell'analisi linguistica del testo sono riconducibili alla nota descrizione di Ferdinand de Saussure che contrappone langue e parole: mentre la prima tende ad individuare un sistema astratto di segni, è la seconda a coglierne l'impatto comunicativo. Non a caso a partire dalla metà del ventesimo secolo l'attenzione è andata a convergere proprio sulla parole, sull'azione comunicativa vera e propria della lingua. L'emergere all'attenzione della parole è infatti alla base della nascita di tutte le discipline che "pongono al centro del loro interesse le caratteristiche generali del prodotto della comunicazione linguistica, cioè il discorso e il testo" (Ferrari 2014: 20). Queste due dimensioni si sono integrate molto velocemente nella prospettiva ipermediale assunta dai nuovi media. Le discipline centrate sul testo, normalmente raggruppate in due aree, quella dell'analisi del discorso e quella dell'analisi linguistica del testo, tendono per loro natura a sovrapporsi e influenzarsi a vicenda, ma nel caso della comunicazione digitale le sovrapposizioni diventano ancora più evidenti e sono coordinate in tempi e modi che dipendono dalla particolare natura diatecnica dei testi multimediali. Analizzando la situazione comunicativa alla fine degli anni novanta del secolo scorso János S. Petőfi affermava di dubitare che fosse possibile parlare di documenti unimediali (cf. Marello/Verdiani c.s.), egli sosteneva che "l'autolimitazione della linguistica basata sul concetto di struttura è resa difficile (per non dire quasi impossibile) dal fatto che gli oggetti della ricerca linguistica sono oggetti il cui aspetto formale/strutturale è soltanto uno fra quelli esistenti, e, dal punto di vista della comunicazione neppure il più rilevante" (Petőfi 1996: 37). Seguendo l'intuizione di Petőfi potremmo dire che "una teoria integrativa (semiotica) degli oggetti che sono parzialmente trattati dalla linguistica" applicata alla Computer Mediated Communication può essere dunque inquadrata solo in un ambito di ricerca interdisciplinare. La dimensione semantica del testo consente di spostare il focus dalla traccia segnata dagli elementi prettamente linguistici del testo alla dimensione inferenziale del medesimo, variamente espressa sul piano diamesico, una dimensione implicita che fa riferimento all'enciclopedia dei parlanti. Il percorso verso la "teoria semiotica della comunicazione umana multimediale" (Petőfi 1996: 34) muove dunque dallo strutturalismo, alla 
linguistica del testo e del discorso, alla pragmatica linguistica integrando via via il nuovo orizzonte multimediale.

\section{Le proprietà di natura pragmatica del testo}

6 La linguistica del testo intesa in senso ristretto tende a concentrare la propria attenzione sugli aspetti prettamente linguistici della comunicazione: la lingua è infatti il codice per eccellenza condiviso dai parlanti, un mezzo che consente di indicare con sicurezza all'altro da noi quale sia la prospettiva corretta in cui leggere un evento comunicativo. L'uso della lingua fa leva su alcune strategie che potremmo definire primarie, cioè su meccanismi di decodifica e inferenza. Come osserva Michele Prandi (2006: 223) l'inferenza va qui intesa come una forma di ragionamento naturale, dunque non una strategia linguistica, ma più generalmente cognitiva. Secondo Ferrari l'inferenza consente in primo luogo di "precisare e completare i significati linguistici espliciti", oltre a questo può però anche "creare contenuti ex novo" (Ferrari 2014, 49). Le inferenze necessarie all'interpretazione di un testo fanno riferimento a diverse categorie di contenuti che possono essere ricavati dal contesto linguistico o cotesto, dal contesto situazionale o dalle conoscenze enciclopediche del parlante. La funzione di decodifica è fondamentale per il riconoscimento del contenuto denotativo del testo e le relazioni strutturali a esso interne. È la lingua infatti a darci le istruzioni di lettura del testo, a fornirci lo schema procedurale rispetto ai contenuti di varia natura semantica in esso presenti. $\mathrm{E}$ una delle procedure per avvicinare la sostanza semantica del testo è appunto l'inferenza che viene formulata a partire dalle informazioni contestuali. Gli atti linguistici non avvengono infatti in una dimensione astratta, ma nella realtà e alla presenza di parlanti diversi, le cui conoscenze sono determinanti per lo svolgimento della conversazione. Come osserva Angela Ferrari «il contesto è dunque il presupposto stesso della comunicazione; esso è inoltre un'entità variabile nella misura in cui muta con l'evolvere della comunicazione e in funzione dei bisogni interpretativi, ed è considerato come condiviso dagli interlocutori; quando si compie un atto comunicativo, si può contare sulla mobilitazione di una conoscenza solo se si sa che l'interlocutore ha o può ricostruire quella conoscenza» (Ferrari 2014: 57). Vista in questa prospettiva l'analisi del testo si presenta dunque molto più 'dinamica' (o procedurale) (Ferrari 2014: 37): il cambiamento di punto di vista sovrappone, in un certo senso, il focus della ricerca all'ambito della pragmatica, il cui obiettivo consiste, come sappiamo, nel descrivere e teorizzare l'uso linguistico in contesto. Per comprendere il testo digitale, oltre che della coerenza e della coesione occorrerà dunque tener conto anche delle altre proprietà di natura pragmatica del testo e in particolare dell'intertestualità.

\section{I paradigmi intertestuali di Gerda Haßler}

7 In un suo saggio del 1997 Texte im Text Gerda Haßler affrontava la questione delle diverse forme linguistiche dell'intertestualità. L'intertestualità costituisce infatti un approccio del quale da tempo ormai sia la cultura artistico letteraria, che la ricerca linguistica non possono non tener conto nell'intento di mettere a fuoco con nitidezza quali siano i modi di leggere i testi nel testo da parte delle diverse comunità scientifiche e di giungere alla formulazione di un approccio teorico unitario e a un concetto di intertestualità univoco ${ }^{4}$. In Texte im Text la linguista mette a fuoco la presenza delle 
citazioni nei testi e i problemi di coerenza e di linearità connessi a questa pratica. Haßler osserva che in alcuni casi la presenza delle citazioni turba la tradizionale sequenzialità lineare dell'uso della lingua. «Il fenomeno dei testi nel testo fa riferimento a interdipendenze e rotture della coerenza, la cui marcatura è altrettanto problematica del contributo che essi possono fornire alla comprensione e quindi alla costituzione stessa del senso del testo» (Haßler 1997b: 11)5. E evidenzia che l'atto di citazione nel mondo moderno porta con sé un implicito atto di riverenza nei confronti dell'autore da cui si cita ${ }^{6}$. Citare implica autorialità ma anche autorità. Chi scrive citando altri autori si ricollega a una tradizione secolare, questo suggerisce che senza la citazione, il continuo ripetere testi non si dia cultura. Ci troviamo di fronte a una citazione testuale quando l'elemento citato è già stato precedentemente parte di una unità di senso. Questa lettura è di centrale importanza per il nostro discorso perché, con l'avvento del digitale, l'uso delle citazioni è diventata una pratica comune, si è svincolata dall'uso letterario o artistico per diventare una delle abitudini più diffuse degli utenti della rete. Citare è infatti una strategia molto compatibile con la velocità di cui la comunicazione in rete si nutre, consente di includere testi di varia natura, spesso multilingui, e viene fruita contemporaneamente da parlanti di lingue diverse. Le citazioni non riguardano solo i testi scritti ma anche quelli visuali e sonori. Anche in questo caso il focus è nella ripetizione di immagini cariche di senso che sono particolarmente pregnanti per il parlante, e anche in questo caso talvolta si generano problemi di coerenza e linearità del senso con l'aggravante di una forte codificazione legata al contesto di provenienza culturale fatto questo che rende non completamente trasparenti i conglomerati finali per gli utenti che provengano da realtà culturali e linguistiche diverse. Se la comunicazione multimodale può dunque essere considerata da sempre la dimensione normale della comunicazione, con i media moderni la sua versatilità emerge ancor più chiaramente, estendendo a tutti gli utenti del web una sorta di dignità autoriale che prima era assente o riservata a una ristretta élite di artisti o intellettuali. In questa prospettiva possiamo parlare di gesto semantico dei testi, “ semantischer Gestus von Texten" (Lachmann 1990: 7; Haßler 1997b: 21), perché le citazioni di testo, di cui un buon esempio d'uso è rintracciabile proprio nella comunicazione politica, sono assimilabili a un gesto deittico e consentono all'emittente di spingere all'estremo l'uso delle ellissi nel testo verbale, il senso da attribuire a un enunciato risulta infatti trasparente solo grazie all'ancoraggio ad immagini particolarmente pregnanti che accompagnano il testo stesso.

Haßler osserva che ogni volta che un elemento citato sia già stato precedentemente parte di una unità di senso, ci troviamo di fronte a un testo nel testo, una struttura di relazioni si costituiscono nei testi come relazioni evidenti e quindi relativamente fisse tra i mezzi lessicali e sono utilizzabili nelle successive produzioni di testi. Per descrivere questa forma di allusione, Haßler introduce il concetto di paradigma intertestuale (Haßler 1997b), cioè una struttura di relazioni costruita a livello testuale, che «attraverso la ripetizione può diventare una norma per un certo tipo di testo, per una tematica, per un soggetto, per la determinazione del punto di vista. Il dato interessante è che l'uso ricorrente dei paradigmi intertestuali determina uno schema di aspettative socialmente normalizzato che renda le relazioni di invarianza e opposizione un fattore costitutivo del significato, che alcuni elementi linguistici presenti nel testo portano già da soli con sé» (Haßler 2019: 37). Nel testo di Amnesty che vedremo nel seguito (7.2) alcune relazioni sintagmatiche espresse dal referente visuale sono stabilizzate e fanno parte di una precisa tradizione, attribuiscono cioè un grande 
potenziale di significato al testo. Come vedremo il richiamo intertestuale a Spartacorealizzato sia con mezzi linguistici che con mezzi visuali anticipa la struttura illocutiva del testo incentrata sulla catena di imperativi collocando un messaggio verbale direttamente all'interno dell'immagine, nella dimensione analogica del testo. La presenza all'interno dell'immagine di un enunciato che è coerente con la struttura testuale della petizione sia sul piano semantico che su quello grammaticale indica che la ricezione dei diversi piani di questo testo avviene in modo integrato, secondo delle strategie di ancoraggio che superano la bipartizione fra parola e immagine.

\section{La dimensione multimediale del testo: la Testologia Semiotica di János S. Petőfi}

La necessità di sviluppare una testologia semiotica secondo János S. Petőfi era appunto legata al fatto che nessuno dei rami della ricerca singolarmente preso fino a quel momento fosse stato in grado di indagare efficacemente «tutti gli aspetti (strettamente interconnessi) della testualità e nessuno di essi è stato concepito in modo tale da integrare i risultati specifici dei rami particolari della ricerca incentrata sul testo» (Petőfi 1985: 371-372; Vitacolonna 1996: 47). Si colloca in questa prospettiva la rilettura del concetto di linguistica del testo proposta da János Petőfi, in essa trova spazio anche la specificità del processo di costruzione del significato legato alla lettura delle immagini e dei messaggi metalinguistici che è una peculiarità della dimensione digitale della comunicazione. L'unità fondamentale della comunicazione non è più secondo Petőfi il testo singolo, ma più testi, compresenti e sovrapposti nell'uso, capaci di costruire il senso di un messaggio attraverso rimandi semiotici interni. Una delle peculiarità di questa categoria di testi complessi è che la lingua, nella forma della scrittura, mantiene un ruolo determinante per la costituzione del significato. János Petőfi, in un saggio del 1990, pubblicato nell'edizione italiana nel 1996, aveva introdotto la nozione di testo prevalentemente verbale: «un testo è un oggetto semiotico relazionale prevalentemente verbale con una manifestazione fisica scritta a mano o stampata» (1996: 67), sostenendo che

[...] un oggetto semiotico relazionale prevalentemente verbale soddisfa i criteri della testualità se vengono soddisfatte le seguenti aspettative; in una data o presunta situazione comunicativa quest'oggetto esprime una configurazione connessa (e completa) di stati di cose e assolve una data o presunta funzione comunicativa; ha una costituzione connessa e completa, laddove la connettezza e completezza della costituzione possono dipendere dal tipo dell'oggetto dato. (Petőfi 1996: 69; mio corsivo).

Partendo dunque dal presupposto che la gran parte dei comunicati sia «multimediale (o unimediale, in modo tale che il medium dato contenga diversi sottocomponenti mediali [...]) e poiché i comunicati multimediali significano in modo etero-mediale, per la loro interpretazione formale e semantica è necessario costruire una disciplina che possa prendere in giusta considerazione questa proprietà» (Petőfi 1996: 118). Petőfi giunge dunque alla formulazione di una disciplina che sia in grado di fornire una descrizione adeguata dei testi multimediali presenti in un testo (cf. Marello/Verdiani c.s.): la Testologia Semiotica. Una disciplina pensata per prendere in considerazione tutti $i$ fattori che giocano un ruolo nella costituzione del significato e che quindi non si limita ad analizzare solo gli aspetti puramente linguistici dei testi, ma li considera nel loro contesto comunicativo multimediale. Il modello della situazione comunicativa letta secondo questa prospettiva è il seguente: 


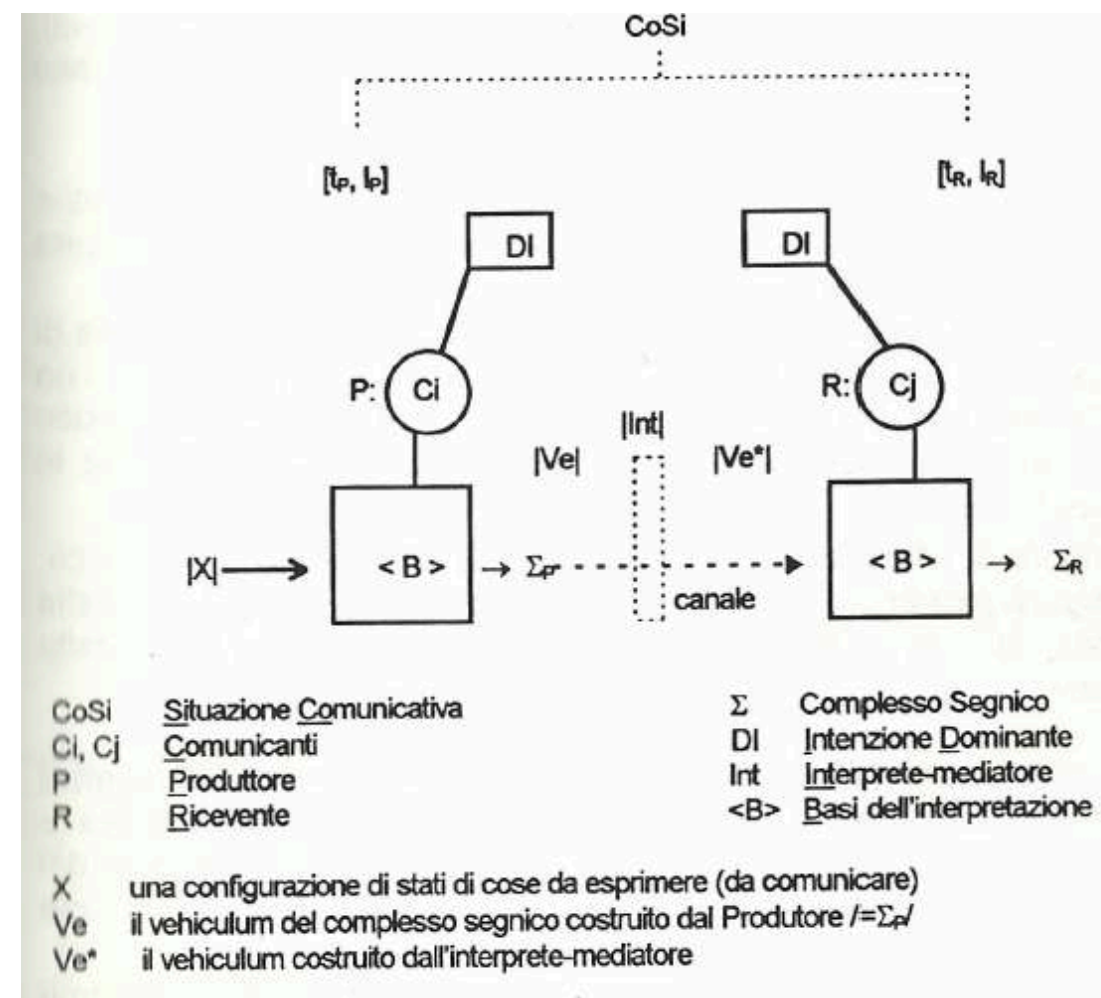

Fig. 2 Fattori costitutivi di una situazione comunicativa (Petőfi 1996, 118).

11 È interessante notare come il modello (Fig. 2) tenga conto della dimensione intenzionale del produttore e dell'interprete e analizzi in modo distinto il vehiculum del complesso segnico costruito dal produttore e il vehiculum costruito dall'interprete mediatore. Il focus di ricerca è in questo caso la dimensione diatecnica e nell'analizzarla si tiene conto dell'inevitabile scarto presente fra la costruzione del produttore e la ricostruzione ermeneutica dell'interprete-mediatore, due dimensioni raramente sovrapponibili.

Negli anni successivi Petőfi ha elaborato la sua Testologia Semiotica, il cui modello è senz'altro influenzato dal rilievo che aveva assunto in quegli anni la società dei media $\mathrm{e}$ che con i dovuti adattamenti sembra adatto a integrare anche i nuovi media del secondo millennio. Al centro dell'elaborazione teorica di Petőfi (Fig. 3) vi è un testo di eterogenea realizzazione $\left(\mathrm{T} \alpha / a / \ldots{ }^{7}\right)$ così come è percepito dall'interprete. Con il modello da lui elaborato è dunque possibile integrare nell'analisi tutti i diversi piani espressivi presenti nei testi ibridi (cf. Marello/Verdiani c.s.). La descrizione grafica e la stringa ideata da Petofi sono aperte e dunque si prestano a essere integrate anche dagli elementi multimediali che caratterizzano attualmente la comunicazione in ambiente digitale. 
/A/

/B/

/C/

/D/

/E/

/F/ /G/

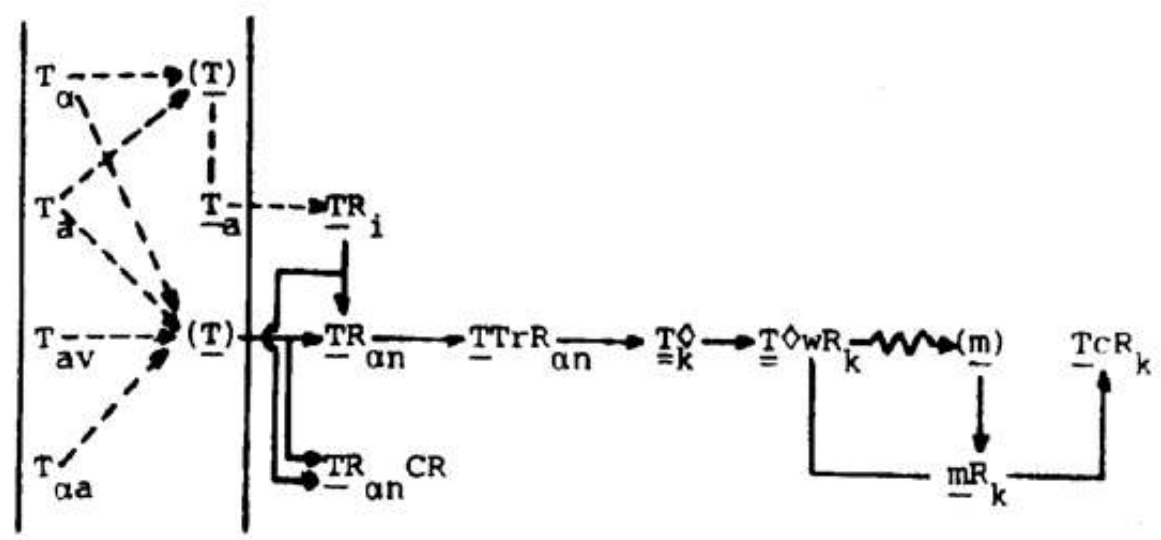

Fig. 3 Elaborazione testuale teorica di J.S. Petôfi (2011:39).

\section{La selezione del contesto}

Nella comunicazione digitale la costruzione del contesto avviene dunque principalmente, ma non esclusivamente sotto la guida della lingua. Il processo di integrazione delle informazioni ottenute dalla decodifica del messaggio linguistico è arricchito e determinato dai dati multimodali e multimediali sulla situazione contestuale che vengono forniti al lettore e che lo mettono nelle condizioni di interagire in modo intuitivo con esso. Il repertorio di immagini (o di altri riferimenti semici extralinguistici) a cui il lettore attinge e che è in grado di riconoscere e interpretare fa riferimento infatti all'enciclopedia che egli è si è creato e che ha caratteristiche comuni al gruppo di interlocutori di cui fa parte. Dunque alla decodifica del messaggio prettamente linguistico, presente nella comunicazione digitale insieme alle immagini, contribuisce l'esegesi di un contesto complesso, a volte molto articolato sul piano culturale. Come evidenzia Angela Ferrari $(2014,81)$ infatti, il testo è un'unità semantica ad alto grado di strutturazione, formato da unità comunicative e relazioni semantiche. Le loro caratteristiche risultano fortemente condizionate dalla natura dei testi presi in esame, che in un certo senso selezionano alcune funzioni prevalenti. Petőfi nella sua Elaborazione testuale teorica tiene conto del rapporto dinamico che si innesca fra i testi da interpretare e la conoscenza del mondo da parte dell'interprete «Nell'interpretare un testo, l'interprete non solo impiega la sua conoscenza del mondo, ma può anche utilizzare il testo per correggere e/o ampliare la sua conoscenza del mondo» (Petőfi 2011, 38).

\section{Le condizioni di coerenza ibride del testo digitale.}

Con l'avvento del testo digitale si modificano radicalmente le condizioni di coerenza testuale, seguendo l'intuizione di Marie-Elisabeth Conte, potremmo segnalare come il focus si sposti dalla coerenza a parte obiecti alla coerenza a parte subiecti e vedere il testo come «una sequenza d'istruzioni la quale guida (pilota) la costruzione della coerenza da parte dell'interprete» (1988: 9). Le condizioni di coerenza dei testi digitali sono legate 
all'atto dell'interprete. Come vedremo negli esempi che seguono la comunicazione online ricorre a diverse strategie che fanno leva su elementi extratestuali per mobilitare le conoscenze degli interlocutori. Spesso si tratta di contenuti che costituiscono un repertorio ad alta densità semantica, trasparente e immediatamente riconoscibile da parte del pubblico perché legato alla tradizione culturale condivisa. Analizzeremo due esempi tratti dalle campagne internazionali di Amnesty International in Italia, Francia, Spagna, Germania, Regno Unito.

\subsection{Campagna mondiale di Amnesty International: Fermiamo la detenzione e la vendita di rifugiati e migranti in Libia}

Il primo caso che prenderemo in analisi è l'azione internazionale di Amnesty International legata alla violazione dei diritti umani in Libia che venne denunciata dalla CNN nell'estate del 2017 con la realizzazione di un reportage diffuso nel novembre dello stesso $a n n o^{8}$. È interessante notare che le due immagini selezionate come copertina delle azioni nelle diverse edizioni (Fig. 4, 5 e 6)- divulgate parallelamente in Gran Bretagna, Germania, Francia, Spagna, Italia sono una citazione del video della CNN, in un certo senso esse collocano il lettore in medias res, richiamano cioè un contesto noto, un evento a cui la stampa ha dato rilievo a livello internazionale, introducendo delle 'immagini chiave', Schlagbilder, (Diekmannshenke 2011: 161) che in questo caso diventano le immagini simbolo dell'azione.

\subsection{Gli effetti sulle scelte linguistiche}

16 I titoli dell'azione nelle diverse lingue sono sostanzialmente sovrapponibili, anche se nella campagna inglese e in quella spagnola sono presenti anche le notazioni urgent/ urgente. In tutte le versioni viene mantenuto l'anglicismo per la forma dell'imperativo, in inglese stop, salvo che in italiano, lingua in cui questa forma è lessicalizzata, ma viene associata meno comunemente a questa forma verbale.

Urgent: stop the selling and detention of refugees and migrants in Libya Urgente: Stop a la detención y venta de personas refugiadas y migrantes en Libia Fermiamo la detenzione e la vendita di rifugiati e migranti in Libia Stop à la détention et à la vente des réfugiés et des migrants en Libye

Per la diffusione internazionale di questa petizione sono state realizzate due diverse copertine. Entrambe le immagini sono attentamente costruite come citazioni dal video della CNN. Nella prima, selezionata per la versione inglese e spagnola della campagna, è visibile un gruppo di uomini seduti per terra davanti a un muro sul quale è chiaramente leggibile una scritta in inglese. La fotografia scelta per la versione inglese e spagnola è la stessa. Il dato interessante è la presenza in essa dell'iscrizione murale To Remember Spartacus (Fig. 4). 


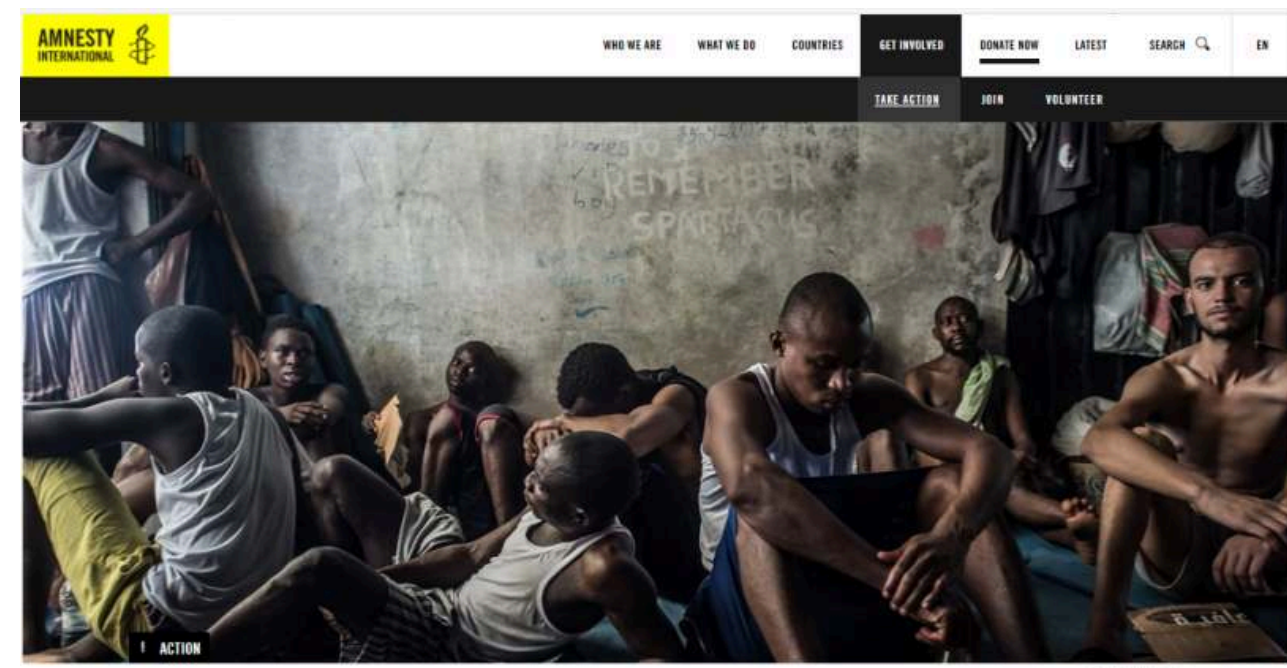

Urgent: stop the selling and detention of refugees and migrants in Libya

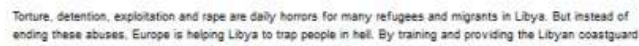

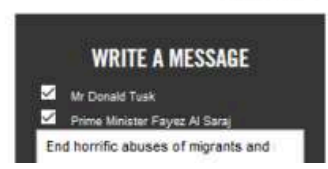

Fig. 4 Amnesty International GB: Urgent: stop the selling and detention of refugees and migrants in Libya $^{9}$

18 La fotografia pubblicata sulla pagina dell'edizione spagnola è stata volontariamente tagliata, la prima parte della frase non è più presente nell'immagine, dell'iscrizione murale rimane leggibile solamente Spartacus (Fig. 5), la parola è comunque ben visibile in primo piano e mantiene la sua efficacia.

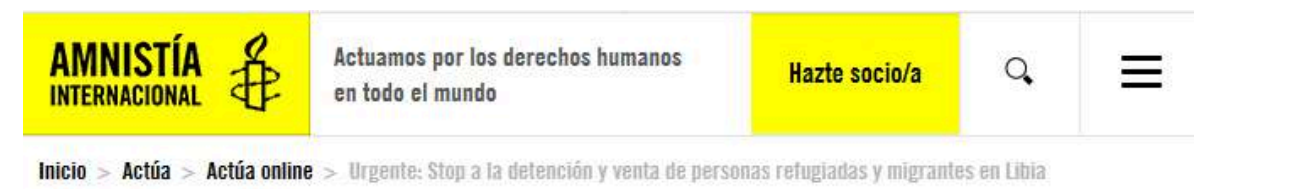

\section{URGENTE: STOP A LA DETENCIÓN Y VENTA DE PERSONAS REFUGIADAS Y MIGRANTES EN LIBIA}

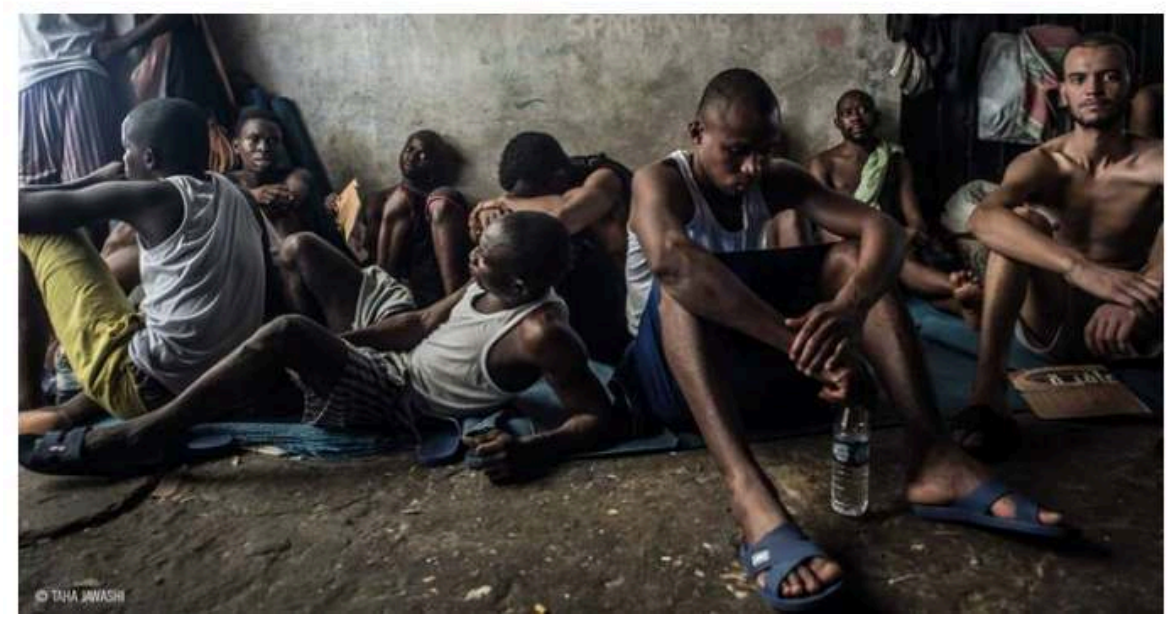

Fig. 5 Amnistia International Spagna. Urgente: Stop a la detención y venta de personas refugiadas y migrantes en Libia ${ }^{10}$. 
In questo secondo caso l'iscrizione To Remember Spartacus in caratteri chiari sul muro grigio che fa da sfondo all'immagine funge da anticipazione per gli utenti della pagina di Amnesty. La scritta è presente in inglese in tutte le versioni. Sia l'immagine che la frase evocano volontariamente il video realizzato della CNN il 14.11.17 e diffuso dalle emittenti internazionali nei giorni successivi. Il loro significato è sovrapponibile all'immagine della gabbia scelta per le azioni italiana e francese (Fig. 6), entrambe le copertine infatti evocano la schiavitù, sono una metafora viva della schiavitù (Prandi 2017). Il riferimento esplicito di questa seconda immagine è alla vicenda dello schiavo romano Spartaco, un personaggio storico vissuto nel I secolo a.C. che appartiene in molti modi all'enciclopedia dei parlanti, e non solo per le numerose riduzioni cinematografiche che hanno garantito la diffusione della sua storia nel corso del tempo. È l'analogia determinata da alcuni dati biografici a giustificare la citazione di Spartaco in questa sede: dopo essere stato catturato dai legionari: Spartaco viene venduto. A comprarlo, secondo quanto scrive Plutarco, è Lentulo Batiato, un piccolo imprenditore che possiede una scuola per gladiatori a Capua, fatto questo che influirà sul suo destino. Nella memoria comune Spartaco non rappresenta però solo la schiavitù, ma anche la ribellione. La scritta: To remember Spartacus a caratteri maiuscoli chiari sul muro grigio alle spalle del gruppo di rifugiati non serve solo alla ricostruzione multimediale del contesto, alla costruzione di un supporto inferenziale corrispondente al profilo culturale medio di un lettore, ma anticipa la struttura testuale illocutiva del testo che segue. Sembra essere un primo punto- dislocato sulla fotografia di una lista di verbi all'infinito di valore finale introdotti dall'imperativo, che costituisce il nucleo illocutivo della petizione, presente alla fine della pagina, non a caso proprio prima del pulsante FIRMA.

To remember Spartacus

Urgent: stop the selling and detention of refugees and migrants in Libya

Send an email now to Libyan and European leaders and urge them to work together

to:

- Release all migrants and refugees from detention centres and end the arbitrary detention of refugees and migrants in Libya

- Investigate all allegations of torture and other ill-treatment of refugees and migrants in Libya, and ensure that the suspected perpetrators are prosecuted in a transparent and fair trial to put an end to the vicious cycle of abuse.

- Review how they cooperate on migration policies, to prioritise protecting the human rights of refugees and migrants, instead of trapping people in Libya

- Formally recognize the UNHCR and allow the organization to carry out its full mandate including the protection of asylum seekers and refugees.

Nella seconda copertina, selezionata per la versione italiana e francese della campagna, è visibile solo un uomo imprigionato dietro a una rete (Fig. 6). 


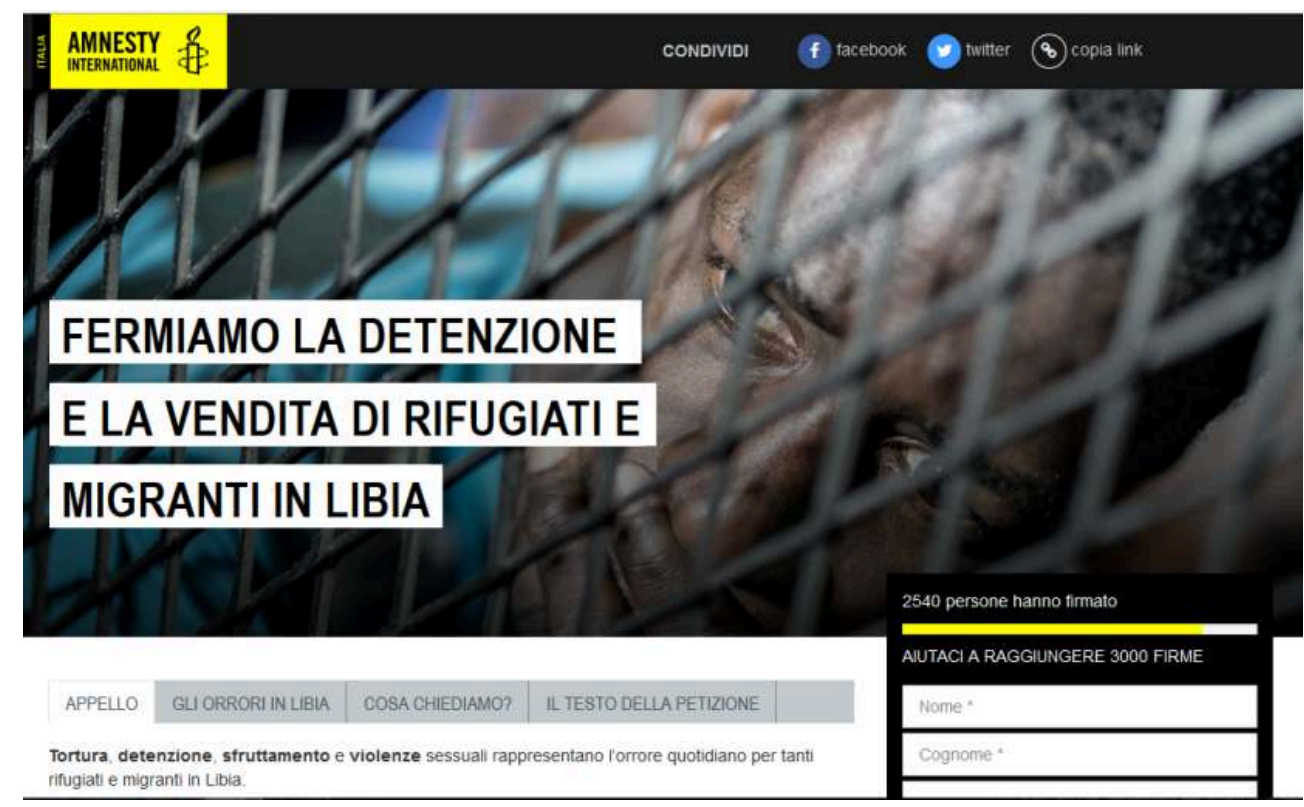

Fig. 6 Amnesty International Italia: Fermiamo la detenzione e la vendita di rifugiati e migranti in Libia'17

21 Sull'immagine campeggia il titolo:

Fermiamo la detenzione e la vendita di rifugiati e migranti in Libia

22 A cui fanno seguito le finalità della petizione secondo uno schema che rispecchia il testo inglese:

Chiediamo ai leader libici ed europei a collaborare urgentemente per:

- rilasciare tempestivamente tutti i migranti e i rifugiati dai centri di detenzione e porre fine alla detenzione arbitraria di rifugiati e migranti in Libia

- indagare su tutte le accuse di tortura e altri maltrattamenti nei confronti di rifugiati e migranti in Libia, e assicurare che i sospetti responsabili siano perseguiti in un processo trasparente ed equo per porre fine al circolo vizioso degli abusi.

- reimpostare la cooperazione con la Libia in materia di migrazione, dando priorità alla protezione dei diritti umani dei rifugiati e dei migranti, invece di intrappolare le persone in Libia

- riconoscere formalmente l'UNHCR in Libia e consentire all'organizzazione di svolgere appieno il suo mandato, compresa la protezione dei richiedenti asilo e dei rifugiati.

In un panorama comunicativo di grande complessità come quello della CMC sembra attuale quanto Marie-Elisabeth Conte osservava alla fine degli anni ‘ 80 :

L'interprete è chiamato a trarre delle inferenze, costruire anelli mancanti, a reinterpretare (in nome della coerenza) segmenti testuali ai quali aveva già assegnato un'interpretazione. Il processo dinamico dell'interpretazione non procede solo linearmente per progressiva accumulazione di informazioni, ma può retroagire (feedback ermeneutico) anche su anteriori interpretazioni di informazioni anteriori, e su anteriori inferenze. (Conte 1988: 10).

24 Nel caso della comunicazione digitale la dimensione intertestuale è preminente e coinvolge molti media contemporaneamente, l'estrema complessità dei testi determina non poche difficoltà di analisi. Difficoltà che sembra possibile affrontare alla luce del panorama teorico che abbiamo qui proposto. 


\section{Conclusioni}

La natura dei testi digitali ha caratteristiche molto particolari: per giungere alla comprensione del messaggio il lettore procede integrando spontaneamente i diversi elementi verbali, visuali e sonori in esso presenti. Come osserva Conte il processo dinamico dell'interpretazione non procede più solo linearmente per progressiva accumulazione di informazioni, ma può retroagire e, nel caso della comunicazione in rete, interagire con gli elementi multimediali presenti nei comunicati. Ogni testo digitale infatti è costruito come un circuito chiuso, in cui la decifrazione del senso del messaggio verbale è inarrivabile se si escludono gli elementi visuali e sonori. Questo ha delle ripercussioni interessanti sulle abitudini espressive dei parlanti in rete, finora descritte solo in modo parziale e limitato ai dati prettamente linguistici. Gli elementi visuali e multimediali presenti all'interno dei comunicati influiscono invece a tutti i livelli sulle produzioni linguistiche in una direzione che potremmo definire di ibridizzazione intertestuale. Come ha evidenziato a più riprese Gerda Haßler l'intertestualità svolge da sempre un ruolo determinante nella costruzione del senso di un testo: se l'identificazione delle singole citazioni non è essenziale alla comprensione, è vero però che individuare i riferimenti intertestuali consente una comprensione più articolata e il riconoscimento delle ambiguità. Al riconoscimento delle ambiguità contribuisce innanzitutto la ripresa dei paradigmi intertestuali (Haßler 1997) che nel caso della comunicazione digitale viene ormai sempre più spesso realizzata attraverso l'impiego di citazioni multimediali in associazione al testo verbale. Il caso analizzato presenta appunto un riferimento intertestuale nella forma ibrida di un conglomerato di lingua e immagine, che si basa sulla relazione stabile fra i singoli lessemi e i singoli elementi dell'immagine. Analizzando testi ibridi di questo genere è possibile collazionare una casistica in cui i riferimenti intertestuali producono degli effetti sulla struttura grammaticale del testo verbale. È quello che accade nelle campagne di Amnesty qui citate, dove il richiamo intertestuale a Spartace realizzato sia con mezzi linguistici che visuali anticipa la struttura illocutiva del testo incentrata sulla catena di imperativi attraverso l'ancoraggio contestuale. In prospettiva digitale la multimedialità sembra dunque interferire con un sistema superspecializzato come quello del linguaggio. Letti in prospettiva iconolinguistica molti dei fenomeni che caratterizzano la comunicazione digitale si prestano a essere descritti facendo ricorso- con le opportune modifiche e aperture - a modelli nati nella prospettiva teorica dell'intertestualità già negli ultimi anni del XX secolo. Nel caso delle campagne di Amnesty, per esempio, il modello proposto nell'Elaborazione testuale teorica di János S. Petőfi, con gli adattamenti dovuti sembrerebbe efficace per la descrizione di casi anche molto complessi sul piano intermediale.

\section{BIBLIOGRAFIA}

BOEHM, Gottfried (1995): Was ist ein Bild? München: Fink. 
BREDEKAMP, Horst (2010): Theorie des Bildakts. Berlin: Suhrkamp Verlag.

CONTE, Maria Elisabeth (1999): Condizioni di coerenza. Ricerche di linguistica testuale. Alessandria: Edizioni dell'Orso.

CONTE, Maria Elisabeth (1977): La linguistica testuale. Milano: Feltrinelli.

COSERIU, Eugenio (2008): Linguistica del testo. Introduzione a una ermeneutica del senso. Roma: Carocci.

COSERIU, Eugenio (1997): Linguistica del testo. Introduzione a una ermeneutica del senso. Roma: Carocci

DIEKMANNSHENKE, Hans-Joachim \& Michael KLEMM \& Hartmut STÖCKL (Hrsg.): (2011): Bildlinguistik.

Theorien. Methoden. Fallbeispiele. Berlin: Erich Schmidt Verlag.

FERRARI, Angela (2014): Linguistica del testo. Principi, fenomeni, strutture. Roma: Carocci editore.

FIORENTINO, Giuliana (2018): "In principio era il dialogo. Verso uno stile dialogico-promozionale

nel web". In: L'italiano e la rete, le reti per l'italiano, a cura di Giuseppe РАTоTA \& Fabio RossI. Firenze:

Accademia della Crusca. GoWare, 2013-2501.

HASSLER, Gerda (1997): “Texte im Text: Überlegungen zu einem textlinguistischen Problem”. In:

Texte im Text. Untersuchungen zur Intertextualität und ihren sprachlichen Formen, hrsg. von Gerda

Haßler. Münster: Nodus Publikationen, 11-58.

HASSLER, Gerda (2019): “L'approccio linguistico all'intertestualità e la sua applicabilità all'analisi intermediale”. In: Intertestualità e Intermedialità. Torino: RiCognizioni, Vol 6, No 12 (2019), 29-51.

HASSLER, Gerda \& Gesina Volkmann (Hrsg.) (2009): Deixis y modalidad en textos narrativos. Münster: Nodus Publikationen.

KJøRUP, Søren (1978): “Pictorial Speech Acts”. In: Erkenntnis 12: 55-71.

MARELlo Carla \& Silvia VERDIANI (c.s.): "La testologia semiotica di J.S. Petőfi: dalla multimedialità alla multimodalità". In: János S. Petőfi in Memoriam. Pescara.

MITCHELL, W. J. T. (2015): Image Science: Iconology, Visual Culture, and Media Aesthetics. Chicago: The University of Chicago Press.

PALERMO, Massimo (2018): “Organizzare il discorso in rete. Caratteristiche della testualità

digitale”. In: L'italiano e la rete, le reti per l'italiano, a cura di Giuseppe PAтоTA \& Fabio Rossi. Firenze: Accademia della Crusca. GoWare, 1591-1975.

PATOTA, Giuseppe \& Fabio ROSSI (2018): “Premessa. L'italiano irretito o una (nuova) rete di varietà". In: L'italiano e la rete, le reti per l'italiano, a cura di Giuseppe PATOTA \& Fabio ROsSI. Firenze: Accademia della Crusca. GoWare, 180-443.

PISTOLESI, Elena (2018): “Storia, lingua e varietà della Comunicazione Mediata dal Computer”. In: L'italiano e la rete, le reti per l'italiano, a cura di Giuseppe PATоTA \& Fabio RossI. Firenze: Accademia della Crusca. GoWare, 443-1083.

PETŐFI, János S. (2011): Per una teoria semiotica integrata del testo. Dalla TeSWeST alla Testologia Semiotica. Lanciano: Carabba.

PETÖFI [sic], János S. \& Luciano VITACOLONNA (eds.) (1996): Sistemi segnici e loro uso nella comunicazione umana. 3. La testologia semiotica e la comunicazione multimediale. Macerata: Università di Macerata.

PRADA, Massimo (2015): L'italiano in rete. Usi e generi della comunicazione mediata tecnicamente. Roma: Franco Angeli.

PRANDI, Michele (2006): Le regole e le scelte. Introduzione alla grammatica italiana. Torino: Utet. 
PRANDI, Michele (2017): Conceptual Conflicts in Metaphors and Figurative Language. New York: Routledge.

SCHMITZ, Ulrich (2003): Blind für Bilder. warum sogar Sprachwissenschaftler auch Bilder betrachten müssen. Duisburg: Laud.

SCHMITZ, Ulrich (2011): „Sehflächenforschung. Eine Einführung“. In: Bildlinguistik. Theorien. Methoden. Fallbeispiele, hrsg. von Hans-Joachim DIEKMANNSHENKE \& Michael KLEMM \& Hartmut STÖCKL. Berlin: Erich Schmidt Verlag, 23-42.

SIEVER, Christina Margrit (2015): Multimodale Kommunikation im Socia7l Web: Forschungsansätze und Analysen zu Text-Bild-Relationen. Frankfurt am Main: Peter Lang.

STÖCKL, Hartmut (2004): Die Sprache im Bild \& das Bild in der Sprache. zur Verknüpfung von Sprache und Bild im massenmedialen Text; Konzepte. Theorien. Analysemethoden. Berlin: de Gruyter Mouton.

TAVoSANIS, Mirko (2011): L'italiano del web. Roma: Carocci.

VERDIANI, Silvia (2019): “Fra lingua e immagini. Introduzione alla linguistica visuale”. In: Borders of the Visible - II: Intersections between Literature and Photography. Torino: «CoSMo | Comparative Studies in Modernism», N. 14 (2019), 257-272.

VERDIANI, Silvia (2016): "Silenzio e immagini". In: Scritture brevi: segni, testi e contesti dalle iscrizioni antiche ai tweet, a cura di Alberto MANCO \& Azzurra MANCINI. Napoli: «Quaderni di Ai $\omega n »$, N.S. 5, 2016, 445-464.

VITAColonNA, Luciano (ed.) (1996): Sistemi segnici e loro uso nella comunicazione umana. 3. La testologia semiotica e la comunicazione multimediale. Macerata: Università di Macerata.

Amnesty Italia: https: \& \& www.amnesty.it \& appelli \& fermiamo-la-detenzione-la-venditarifugiati-migranti-libia \& (ultimo accesso: 25.12.2018).

Amnesty Francia: https: \& \& www.amnesty.fr \& refugies-et-migrants \& petitions \& stop-a-ladetention-et-vente-des-refugies-et-migrants-en-libye (ultimo accesso: 25.12.2018).

Amnesty Spagna: https: \& \& www.es.amnesty.org \& actua \& acciones \& libia-venta-refugiadosmigrantes-dic17 \& (ultimo accesso: 25.12.2018).

Amnesty GB: https: \& \& www.amnesty.org \& en \& get-involved \& take-action \& urgent-stopselling-and-detention-of-refugees-and-migrants-in-libya \& (ultimo accesso: 25.12.2018).

Amnesty Germania: https: \& \& www.amnesty.de \& informieren \& aktuell \& libyen-inakzeptablemigrationspolitik-traegt-zu-schwersten (ultimo accesso: 25.12.2018).

\section{NOTE}

1. Articolata in diverse aree di ricerca liminari: Visual Studies (Mitchell 2015; Boehm 1994), della iconolinguistica o Bildlinguistik (Schmitz 2003, 2011; Stöckl 2004; Diekmannshenke/Klemm/Stöckl 2011; Siever 2015), della testologia semiotica (Petőfi 1996) e della teoria dell'atto iconico (Kjørup 1978; Bredekamp 2015).

2. Patota \& Rossi 2018. Cfr. anche Palermo 2018; Fiorentino, 2018; Lubello 2017; Antonelli, 2016; Prada, 2015; Pistolesi, 2014.

3. Per l'analisi dell'evoluzione terminologica di ambito iconolinguistico cf. Siever 2015.

4. Die Intuition, dass Texte ihren Sinn aufgrund eines Zusammenwirkens von Perspektiven und Stimmen konstituieren, hat bereits von ihrer konzeptuellen Fixierung im Begriff der Intertextualität Wissenschaftstraditionen geprägt. (Haßler 1997b: 7). 
5. Das Phänomen der Texte im Text verweis auf Abhängigkeiten uns Kohärenzbrüche, deren Markiertheit ebenso problematisch ist wie ihr Beitrag zur Verstehensvorgabe des Textes und damit zur Konstituierung des Textsinns (1997b: 11).

6. Come ricorda Haßler il termine francese medievale Respit sentenza è la continuazione del latino respectus, secondo l'etimo individuato da Lisa Block de Behar (1995: 63) cit. in Haßler (1997b: 11).

7. T $\alpha$ : un testo della lingua-oggetto in forma scritta (a: alfabetico); Ta : un testo della linguaoggetto in forma orale (a: auditivo) ; Ta : un testo della lingua-oggetto contemporaneamente letto e ascoltato ; Tav : un testo audio-visivo della lingua-oggetto ; $(\underline{T}):$ un $\mathrm{T} \alpha / \alpha / \ldots$ così come è percepito da un interprete (inaccessibile a un'analisi intersoggettiva) ; ( $\underline{\mathbf{T}}) \mathbf{L}:$ un $\mathrm{T} \alpha / a / \ldots$ Le azioni necessarie alla lettura (L: lettura); $\underline{T} \mathbf{a}$ : un $\underline{\mathrm{T}}$ proferito o letto dall'interprete ; $\underline{\text { Tri }}$ : la registrazione acustico-fonetica di un $\underline{T}$ a (i: strumentale) ; $\underline{T} \boldsymbol{\alpha} \alpha \mathbf{n}$ : una $\underline{T} \mathrm{Ri}$ trasformata, rappresentata nei termini di una lingua-oggetto normalizzata; oppure un $\underline{T}$ rappresentato dall'interprete ; Tr $\alpha \mathbf{C R}$ : la rappresentazione dei commenti relativi alla costruzione della $\underline{T} R \alpha n$; TTrRon : una TR $\alpha$ n disambiguata e/o integrata, rappresentata nei termini della stessa linguaoggetto normalizzata impiegata per la rappresentazione della $\underline{T} \alpha \propto n ; \underline{T}^{0} \mathbf{k}$ : la rappresentazione canonica di una $\underline{\mathrm{TT}} R \alpha \mathbf{n} ; \underline{\mathbf{T}}^{\mathbf{w}} \mathbf{w R k}$ : la rappresentazione canonica dell'ipotesi comunicativa dell'interprete relativa al rapporto fra gli stati di cose rappresentati in una $\left.\underline{T}^{\ominus} \mathrm{k} ; \underline{\mathbf{m}}\right)$ : un modello (un insieme di modelli) che contiene un complesso di stati di cose reali o fittizi, attivato nella memoria/immaginazione dell'interprete da una $\underline{T}^{\ominus} \mathrm{WRk}$ o costruito in relazione ad essa (inaccessibile a un'analisi intersoggettiva). (Petőfi 2011: 39).

8. La data scelta per la pubblicazione del reportage 14.11 .17 corrisponde a quella della denuncia del caso da parte dell'ONU alla UE. "Aste per vendere migranti come schiavi. Succede in Libia, secondo quanto denuncia la $\mathrm{Cnn}$ in un servizio esclusivo dal titolo Persone in vendita. Dove le vite vengono messe all'asta per 400 dollari. Nel giorno in cui l'Onu accusa l'Ue per l'accordo con la Libia mirato a fermare gli arrivi in Europa, parlando di «orrori inimmaginabili» nei centri libici di detenzione per migranti, l'emittente americana rivela l'esistenza di questo tipo di aste in almeno nove località libiche e pubblica un video shock: il banditore d'asta, di cui nel filmato si vede soltanto la mano posizionata su uno degli uomini in vendita, un nigeriano di circa 20 anni, presenta agli acquirenti un gruppo di persone in vendita come «ragazzi grandi e forti per lavori agricoli». «Ottocento, 900, mille, 1.100», venduto per 1.200 dinari libici, dice il banditore; l'equivalente di 800 dollari. » (La stampa online).

9. https://www.amnesty.org/en/get-involved/take-action/urgent-stop-selling-and-detentionof-refugees-and-migrants-in-libya/ (ultimo accesso: 25.12.2018).

10. https: \& \& www.es.amnesty.org \& actua \& acciones \& libia-venta-refugiados-migrantes-dic17 (ultimo accesso: 25.12.2018).

11. https://www.amnesty.it/appelli/fermiamo-la-detenzione-la-vendita-rifugiati-migranti-libia/ (ultimo accesso: 25.12.2018).

\section{RIASSUNTI}

La funzione indessicale riveste ruolo cruciale nell'uso del linguaggio verbale su Internet che è associato alla presenza di un archivio di riferimento condiviso e determina un nuovo tipo di inferenzialità nel destinatario. Nella comunicazione in rete il significato di una frase è dato 
infatti dall'effetto di diverse percezioni, si tratta in sostanza di comunicati di tipo ibrido, conglomerati di lingua e immagine (Schmitz 2003), caratterizzati dalla presenza di una relazione stabile fra singoli lessemi e singoli elementi iconici. Ci troviamo dunque di fronte a un processo di ibridazione in cui la metamorfosi dei generi e delle strutture testuali assume di per sé un peso semantico e costituisce un valore per gli interlocutori. Internet e in particolare i social media consentono di osservare questi conglomerati di linguaggio e immagine mentre i parlanti li creano, per questo, con l'avvento della cultura digitale, essi sono diventati l'oggetto di uno specifico campo di studi che fa riferimento alla linguistica del testo, linguistica multimodale e all'iconolinguistica, Bildlinguistik. A sostenere questa prospettiva di ricerca contribuiscono tuttavia già alcuni approcci teorici precedenti che discuteremo in questo contributo, per esempio la direzione di ricerca incentrata sull'analisi linguistica dell'intertestualità di Gerda Haßler o la descrizione della comunicazione multimediale di János S. Petőfi.

The indexical function of language has a crucial role in the use of verbal language on the Internet which is associated with the presence of a shared archive of reference and determines a new kind of inferentiality in the receiver. In network communication, the meaning of a sentence seems to be given by the effect of different perceptions that are all simultaneously present in the utterance: this effect is achieved by using conglomerates of language and image (Schmitz 2003). The metamorphosis of the online communication structure made it possible to analyse more clearly the model of these conglomerates. Internet, especially social media, in fact, allows us to observe messages while speakers produce them; they have become the object of a specific field of study, and refer both to Text Linguistics, Multimodality and, in the German, area to Bildlinguistik. However, some previous theoretical approaches that will be discussed in this contribution also contribute to supporting this research perspective, for example the research direction focused on the linguistic analysis of intertextuality by Gerda Haßler or the description of multimedia communication by János S. Petőfi.

\section{INDICE}

Keywords : Text Linguistics, Multimodal Linguistics, Iconolinguistics, Semiotic Testology

\section{AUTORE}

\section{SILVIA VERDIANI}

Universität Potsdam, Università di Torino, Università di Genova 